\title{
1.3. ANÁLISE MULTIVARIADA DA SOJA CULTIVADA SOB SISTEMA PLANTIO DIRETO EM COBERTURAS VEGETAIS DE MILHETO E SORGO
}

\author{
BIOENG $\quad$ B. C. Góes ${ }^{1,2 *}$, R. J. Goes ${ }^{3}$, C. P. Cremasco ${ }^{1}$, L. R. A. Gabriel Filho1
}

\author{
${ }^{1}$ Universidade Estadual Paulista (UNESP), Faculdade de Ciências e Engenharia, Campus de \\ Tupã - SP, Brasil \\ ${ }^{2}$ Universidade José do Rosário Vellano (UNIFENAS), Faculdade de Agronomia, Campus de \\ Alfenas-MG, Brasil \\ ${ }^{3}$ Universidade Federal de Goiás (UFG), Faculdade de Agronomia, Campus de Goiânia-GO, \\ Brasil
}

Article history: Received 17 September 2020; Received in revised form 18 September 2020; Accepted 18 September 2020; Available online 30 September 2020.

\section{RESUMO}

A cultura da soja é a principal commodity do agronegócio brasileiro, sendo o país considerado o maior produtor e exportador da cultura no mundo no ano de 2019, sendo cultivada em grande parte na região Centro-Oeste e Sul do país. Nas últimas décadas, o manejo da cultura vem se intensificando sob o sistema plantio direto, aproveitando a palhada de culturas de coberturas como forma de nutrientes para o solo e aproveitados pela cultura sucessora, a soja, sob o qual, tal sistema de plantio já é adotado em quase $90 \%$ das plantações de soja no Brasil. Nesse sentido, é comum a utilização de fertilizantes nitrogenados, visando na maior produção de matéria, revigorando os nutrientes do solo, disponíveis para a cultura da soja, com incremento do molibdênio via foliar, para auxiliar na absorção do nitrogênio por parte das culturas fixadoras de nitrogênio. Dessa maneira, foi realizada a análise multivariada de variáveis de produtividade, biométricas e nutricionais da cultura da soja cultivada sob plantio direto em coberturas vegetais de milheto e sorgo, com o propósito de identificar o relacionamento de tais nutrientes com o desempenho produtivo da cultura da soja. Para tal, verificou-se que determinados nutrientes, como potássio e nitrogênio, se correlacionam com variáveis indicadoras de produtividade.

Palavras-chave: Produtividade. Variáveis Biométricas. Variáveis Nutricionais.

\section{MULTIVARIATE ANALYSIS OF SOY CULTIVATED UNDER NO-TILLING SYSTEMS IN PLANT COVERS OF MILET AND SORGHUM}

\begin{abstract}
The soybean crop is the main commodity of Brazilian agribusiness, being the country considered the largest producer and exporter of the crop in the world in 2019, and it is cultivated largely in the Midwest and South regions of the country. In the last decades, crop management has been intensified under the no-tillage system, taking advantage of mulch crops as a form of nutrients for the soil and benefited by the successor crop, soybean, under which such planting system is already adopted in almost $90 \%$ of soy plantations in Brazil. In this sense, it is common to use nitrogen fertilizers, aiming at greater production of matter, invigorating the soil nutrients, available for soybean cultivation, with an increase in
\end{abstract}

*bruno.goes@unifenas.br 
molybdenum via leaf, to assist in the absorption of nitrogen by the fixing cultures of nitrogen. In this way, a multivariate analysis of productivity, biometric and nutritional variables of soybean cultivated under no-tillage in vegetable coverings of millet and sorghum was carried out, with the purpose of identifying the relationship of such nutrients with the productive performance of soybean culture. To this end, it was found that certain nutrients, such as potassium and nitrogen, correlate with variables that indicate productivity.

Keywords: Productivity. Biometric Variables. Nutritional Variables.

\section{INTRODUÇÃO}

O setor agrícola tem grande importância na economia brasileira, influenciado pelo aumento populacional e pela crescente demanda de alimentos, tanto em quantidade como em qualidade. A cultura da soja (Glycine $\max$ (L.) Merrill) é caracterizada por ser uma das principais commodities do país, sendo seu uso destinado à alimentação humana e animal, como também à produção de energia (SANTOS; CAMPOS, 2020).

A soja é uma cultura de origem asiática, de regiões da China antiga, que se expandiu rapidamente para outras regiões ao redor do mundo, chegando ao Brasil em meados do século XIX, na região nordeste do país (ALMEIDA JÚNIOR et al., 2020).

A boa adaptação da cultura da soja ao solo e clima brasileiro, fez com que o país se tornasse um dos principais produtores e exportadores da commodity no mundo, destacando-se a região do Centro-Oeste como maior produtora da cultura no Brasil (SILVA et al., 2018; SILVA; NETO, 2019).

Nesse sentido, o setor de produção agrícola busca a inserção de novos mecanismos que possam assegurar altos níveis de produtividade, de modo a manter e atender a demanda do mercado consumidor, ao mesmo tempo que se preza pela preservação e conservação dos recursos naturais, introduzindo novos conceitos e tecnologias para conservação dos recursos (PARDO; MARTÍNEZ, 2019).

A agricultura conservacionista surgiu no início da década de 1990, com o intuito de auxiliar na fertilidade do solo, alterando minimamente a sua estrutura e a biodiversidade, por meio da introdução de princípios, como: mínimo revolvimento mecânico do solo, rotação de culturas e adoção de coberturas vegetais, por meio do Sistema Plantio Direto (SPD) (ARATANI; MADALOSSO; POMPEU, 2018).

O SPD é uma ferramenta do conceito da agricultura conservacionista, que abrange técnicas de manejo do solo, que busca melhorar e conservar o ambiente de cultivo e ao mesmo tempo aumentar a produtividade da cultura, proporcionando melhorias nas propriedades físicas, químicas e biológicas do solo, em virtude pela manutenção da palhada de cobertura vegetal (FAGUNDES et al., 2019).

As coberturas vegetais apresentam grande importância para as culturas em sucessão, pois favorecem a fertilidade do solo pela decomposição da matéria orgânica, a ciclagem de nutrientes, além de aumentar a retenção de água no solo e auxiliar no combate a erosões (SILVA et al, 2017).

A adoção de coberturas vegetais adequadas é de fundamental importância para recuperação da capacidade produtiva do solo. Dentre elas o milheto e o sorgo, tem mostrado maior potencial de sustentação dessas características; quanto a maior produção de matéria orgânica, reciclagem de nutrientes, proteção do solo contra agentes climáticos, além de apresentarem raízes profundas e resistentes a estes fatores (ROSSI et al., 2013; BIESDORF, et al., 2018).

Diante dos avanços e vantagens em torno deste conceito, o SPD tem se mostrado bastante difundido na cultura da soja, principalmente pela sua boa adaptação, sendo adotado na grande maioria das plantações de soja no Brasil (PASSOS et al., 2015).

A cultura da soja apresenta suscetibilidade em sintetizar nitrogênio por associação simbiótica, na qual, em situações de 
deficiência nutricional as plantas podem apresentar-se com má formação dos tecidos vegetativos, atrofiamento das folhas, e consequente queda de produtiva (SCHNEIDER, et al., 2017).

A fixação biológica de nitrogênio na soja pode ser comprometida pela deficiência de molibdênio (Mo), sendo importante buscar técnicas que facilitem sua fixação. Dentre elas, a aplicação de Mo via foliar na planta, permite melhor absorção, devido a sua mobilidade vegetal, que, muitas vezes, não é assegurada quando realizada via solo (BERNARDES; ORIOLI JUNIOR, ARAUJO, 2019).

Portanto, o estudo da disponibilidade de nutrientes em um solo e a correlação com a produtividade, permite o desenvolvimento de tomada de decisões que serão aplicadas nas culturas, principalmente quanto a otimização de calagem e adubação, além da seleção de um manejo mais adequado para cada situação (PEREIRA et al., 2016).

\section{MATERIAIS E MÉTODOS}

O experimento foi realizado nos anos agrícolas de 2013/14 e 2014/15, na cidade de Selvíria-MS, na Fazenda de Ensino Pesquisa e Extensão (FEPE) pertencente à Universidade Estadual Paulista "Júlio de Mesquita Filho" (UNESP) de Ilha Solteira. Sua posição geográfica, localiza-se nas coordenadas de longitude Oeste $51^{\circ} 22^{\prime}$ e de latitude Sul $20^{\circ} 22^{\prime}$, com altitude de 335 metros e solo classificado como Latossolo Vermelho de textura argilosa (GOES, 2016; SANTOS et al., 2018). O clima da região, segundo a classificação Köppen, é de tipo Aw com características de precipitação total anual média de 1.330 mm com umidade relativa do ar em $66 \%$ e temperatura média de $25^{\circ} \mathrm{C}$ (CENTURION, 1982).

Foi realizada a análise multivariada das variáveis estudadas para verificar o grau de relacionamento entre as mesmas, a fim de determinar o grau de similaridade e dissimilaridade entre as mesmas em relação aos diferentes níveis de nitrogênio e molibdênio.
A aplicação de métodos estatísticos multivariados para o estudo da disponibilidade de nutrientes em um solo, demonstram ser uma boa ferramenta para auxiliar o produtor nas tomadas de decisões. A análise multivariada permite reunir em pequenos grupos, um elevado número de dados e informações, de modo a compreender o comportamento e a existência de dependência entre essas variáveis, sendo comum o uso em estudos na área agronômica (HONGYU; SANDANIELO; JUNIOR, 2016; STRECK et al., 2017; LOPES et al., 2018).

Dante do exposto, este trabalho buscou desenvolver uma análise multivariada em relação ao comportamento das variáveis biométricas e nutricionais na cultura a soja, com avaliação nas interações das variáveis em relação à diferentes doses de molibdênio aplicado via foliar e nitrogênio como fertilizante nas coberturas vegetais.

Foram analisadas variáveis como além da produtividade (PRO), as variáveis biométricas: estande (EST) [mil plantas ha ${ }^{-}$ ${ }^{1}$ ], altura de inserção da primeira vagem (AIV) $[\mathrm{cm}]$, altura da planta (APL) $[\mathrm{cm}]$, número de vagens por planta (NVP), número de grãos por planta (NGP), número de grãos por vagem (NGV) e massa de 1000 grãos (MG) [g]; e variáveis nutricionais das folhas, sendo elas: os teores de nitrogênio (TN) $\left[\mathrm{g} \cdot \mathrm{kg}^{-1}\right]$, fósforo (TP) $\left[\mathrm{g}_{\mathrm{kg}} \mathrm{kg}^{-1}\right]$, potássio (TK) $\left[\mathrm{g} \cdot \mathrm{kg}^{-1}\right]$, cálcio (TCa) $\left[\mathrm{g} . \mathrm{kg}^{-1}\right]$, magnésio (TMg) $\left[\mathrm{g} \cdot \mathrm{kg}^{-1}\right]$, enxofre (TS) $\left[{\mathrm{g} . \mathrm{kg}^{-1}}^{-1}\right.$; cobre (TCu) $\left[\mathrm{mg} . \mathrm{kg}^{-}\right.$ $\left.{ }^{1}\right]$; zinco (TZn) [mg. $\left.\mathrm{kg}^{-1}\right]$; manganês (TMn) $\left[\mathrm{mg}^{\mathrm{kg}} \mathrm{kg}^{-1}\right]$ e molibdênio (TMo) $\left[\mathrm{mg} . \mathrm{kg}^{-1}\right] ; \quad$ totalizando 18 variáveis estudadas.

Para avaliação do teor foliar dos nutrientes, foi realizada a coleta das folhas no momento da expansão do terceiro trifólio durante o estádio R2 em 30 plantas por parcela, sendo posteriormente, as amostras levadas à estufa de circulação forçada por um período de 72 horas à $65^{\circ} \mathrm{C}$ 
e moídas no moinho do tipo Wiley (RAIJ et al., 1996; GOES, 2016).

$\mathrm{O}$ estande foi determinado por meio da contagem das plantas contidas em $3 \mathrm{~m}$ de comprimento nas três linhas centrais, realizando a conversão do número de plantas por hectare.

A altura de inserção foi realizada medindo a distância da inserção da primeira vagem em relação ao colo da planta, já a altura da planta foi calculada com base no colo até o ápice da haste principal em dez plantas por cada parcela.

A massa de 1000 grãos foi obtida pela pesagem de uma amostra contendo 1000 grãos de cada parcela em uma balança de precisão $(0,01 \mathrm{~g})$, sendo posteriormente determinada a umidade dos grãos pelo método da estufa $\left(105 \pm 3^{\circ} \mathrm{C}\right)$ com uma correção de $13 \%$ para a massa obtida, na qual realizou a conversão para área útil da parcela para determinação da produtividade dos grãos (GOES, 2016).

A utilização da técnica de análise de dados multivariada permite estimar a

\section{RESULTADOS E DISCUSSÃO}

Foi elaborada a análise multivariada das variáveis de saída da cultura da soja em relação a cobertura vegetal de milheto para os anos agrícolas de 2013/14 e 2014/15, por meio da correlação de importância das variáveis em relação ao processo de classificação de dados, identificando as variáveis que prejudicam o desempenho do modelo (CHANDRASHEKAR; SAHIN, 2014).

Desta maneira, se estabelece a relação entre as variáveis, formando grupos, conhecidos como clusters, para elaboração do dendrograma (FORINA et al., 2007; CHONG; LEE; LING, 2013).

O grau de similaridade é calculado com base na distância entre as variáveis, estando diretamente relacionada ao grau de correlação entre as mesmas. A exemplo, se tivermos um grau de correlação entre duas variáveis de 0,879 , sua distância no dendrograma, será calculada por meio da subtração de uma unidade, ou seja, 1 $0,879=0,121$, ou seja, quanto maior for o grau de correlação entre as variáveis, menor será seu distanciamento na apresentação gráfica do dendrograma.

Pearson com significância de 5\%. Os níveis de similaridade entre as variáveis, estão representados nas Figuras 1 e 2, por meio de dendrogramas.

Figura 1. Dendrograma das variáveis de saída da cultura da soja, do ano agrícola 2013/14 para a cobertura vegetal de milheto.

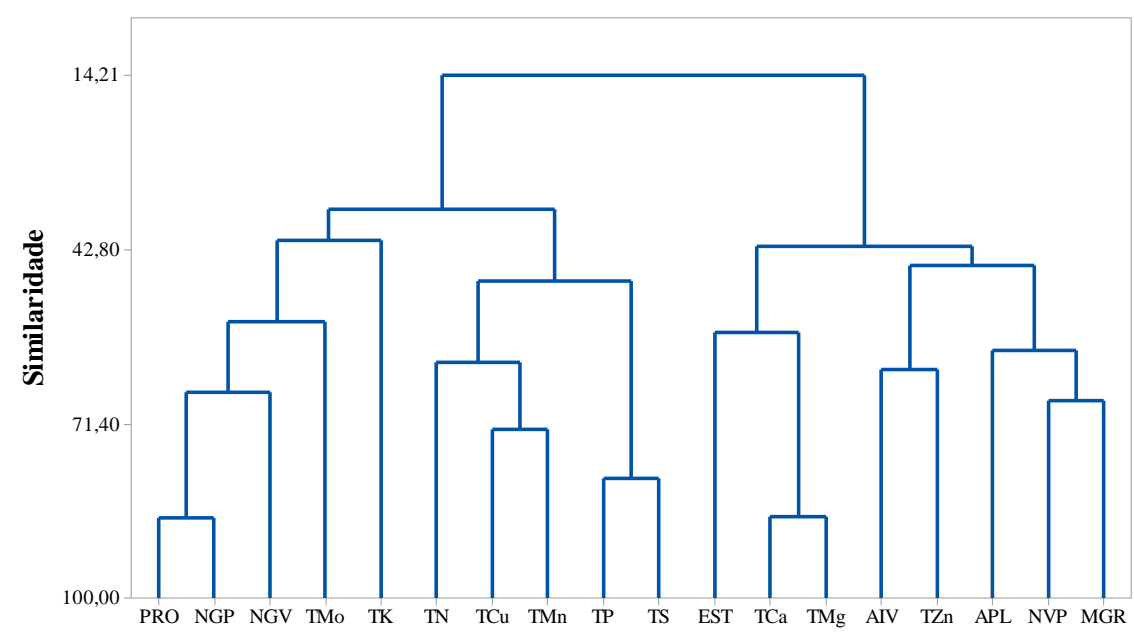

Fonte: Elaborada pelos autores. 
Figura 2. Dendrograma das variáveis de saída da cultura da soja, do ano agrícola 2014/15 para a cobertura vegetal de milheto.

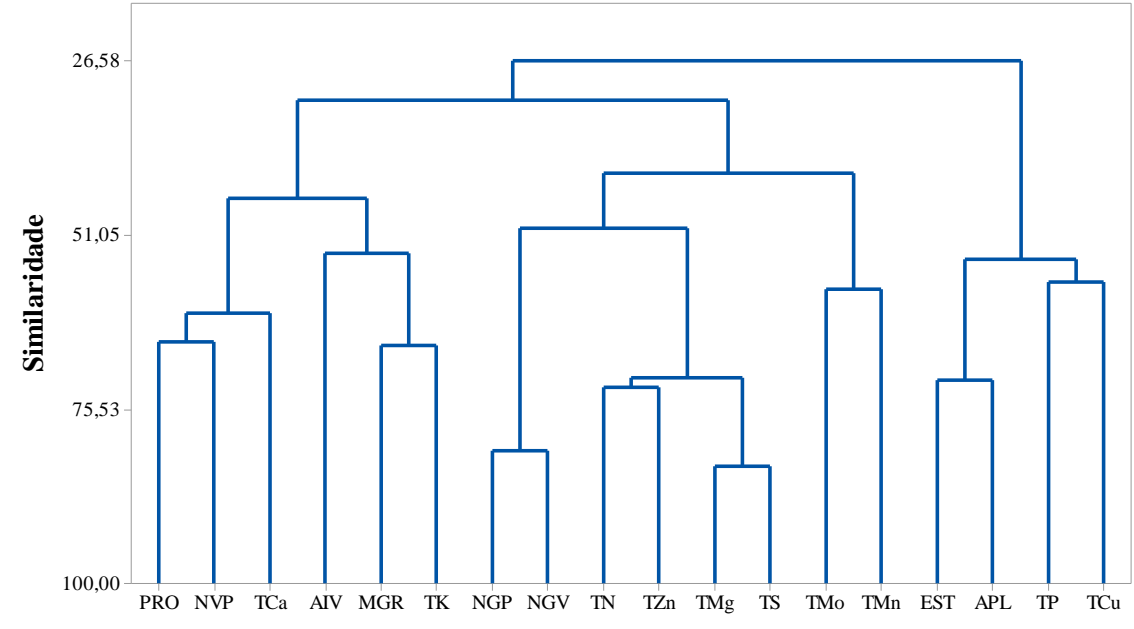

Fonte: Elaborada pelos autores.

A análise gráfica do dendrograma representa a formação de grupos de variáveis com níveis de similaridade em relação ao comportamento frente às diferentes dosagens de nitrogênio e molibdênio que foram utilizadas no experimento. Ao passo que, quanto maior o grau de similaridade, menor será o número de variáveis agrupadas, ocasionando na formação de um maior número de grupos; do mesmo modo, ao passo que diminui o grau de similaridade, ocorre a formação de números menores de grupos, porém com maior quantidade de variáveis agrupadas.

Sendo assim, em relação ao primeiro ano do experimento, nota-se alta correlação entre as variáveis de produtividade, número de grãos por planta e número de grãos por vagem, presentes no primeiro cluster, classificado por apresentarem comportamentos semelhantes, como também apresentado no experimento de Vitorino (2014), no qual, sob efeito de bioestimulantes, as variáveis biométricas: o número de galhos, matéria seca e altura da planta, obtiveram mesmo comportamento, nesse caso, um aumento nos valores sob efeito da cinetina. Assim como também, verifica-se o teor de nitrogênio e potássio próximos essas três variáveis, influenciando diretamente do desempenho produtivo da cultura da soja cultivada sob a palhada do milheto.

Para análise complementar do dendrograma, são apresentados os passos de Amalgamação na Tabela 6, demonstrando o número de variáveis agrupadas a cada passo a nível de similaridade. A distância entre os grupos formados, caracteriza a relevância quanto à quantidade de cluster necessários para o agrupamento, a medida em que ocorre uma mudança abrupta entre eles. 
Tabela 1. Passos de amalgamação das variáveis de saída da cultura da soja para a cobertura vegetal de milheto.

\begin{tabular}{c|ccc|c|ccc}
\hline \multicolumn{5}{c|}{$\mathbf{2 0 1 3 / 1 4}$} & \multicolumn{3}{|c}{$\mathbf{2 0 1 4 / 1 5}$} \\
\hline Passo & Agrupados & Similaridade & Distância & Passo & Agrupados & Similaridade & Distância \\
\hline $\mathbf{1}$ & 17 & 86,8541 & 0,26292 & $\mathbf{1}$ & 17 & 83,4005 & 0,33199 \\
$\mathbf{2}$ & 16 & 86,487 & 0,27026 & $\mathbf{2}$ & 16 & 81,3398 & 0,37320 \\
$\mathbf{3}$ & 15 & 80,2842 & 0,39432 & $\mathbf{3}$ & 15 & 72,3384 & 0,55323 \\
$\mathbf{4}$ & 14 & 72,2339 & 0,55532 & $\mathbf{4}$ & 14 & 71,3339 & 0,57332 \\
$\mathbf{5}$ & 13 & 67,5029 & 0,64994 & $\mathbf{5}$ & 13 & 71,0792 & 0,57842 \\
$\mathbf{6}$ & 12 & 66,2213 & 0,67557 & $\mathbf{6}$ & 12 & 66,4607 & 0,67079 \\
$\mathbf{7}$ & 11 & 62,4889 & 0,75022 & $\mathbf{7}$ & 11 & 65,9711 & 0,68058 \\
$\mathbf{8}$ & 10 & 61,2941 & 0,77412 & $\mathbf{8}$ & 10 & 61,9308 & 0,76138 \\
$\mathbf{9}$ & 9 & 59,2616 & 0,81477 & $\mathbf{9}$ & 9 & 58,6552 & 0,82690 \\
$\mathbf{1 0}$ & 8 & 56,2753 & 0,87449 & $\mathbf{1 0}$ & 8 & 57,6962 & 0,84608 \\
$\mathbf{1 1}$ & 7 & 54,6035 & 0,90793 & $\mathbf{1 1}$ & 7 & 54,3459 & 0,91308 \\
$\mathbf{1 2}$ & 6 & 47,8714 & 1,04257 & $\mathbf{1 2}$ & 6 & 53,5411 & 0,92918 \\
$\mathbf{1 3}$ & 5 & 45,3622 & 1,09276 & $\mathbf{1 3}$ & 5 & 50,0942 & 0,99812 \\
$\mathbf{1 4}$ & 4 & 42,2046 & 1,15591 & $\mathbf{1 4}$ & 4 & 45,8792 & 1,08242 \\
$\mathbf{1 5}$ & 3 & 41,1878 & 1,17624 & $\mathbf{1 5}$ & 3 & 42,2617 & 1,15477 \\
$\mathbf{1 6}$ & 2 & 36,1375 & 1,27725 & $\mathbf{1 6}$ & 2 & 32,1815 & 1,35637 \\
$\mathbf{1 7}$ & 1 & 14,2055 & 1,71589 & $\mathbf{1 7}$ & 1 & 26,5777 & 1,46845 \\
\hline
\end{tabular}

Fonte: Elaborada pelos autores

É possível observar na Tabela 1, o aumento relativo da distância entre os agrupamentos formados ao longo de cada passo. Por exemplo, para o ano 2013/14, entre o passo 1 e 16 nota-se um aumento médio de 0,07 a cada passo, já quando ocorre o avanço de 16 para o 17, nota-se uma mudança abrupta da distância, de aproximadamente 0,44 , o que pode representar a região à ser seccionada no dendrograma, identificando a quantidade necessária de cluster, para um nível de similaridade de $36,13 \%$ entre as variáveis.

Já em relação ao ano 2014/15, notase uma mudança abrupta entre o passo $15 \mathrm{e}$
16, para uma formação de 3 clusters, com similaridade de 42,26\% entre as variáveis.

Por sua vez, a distância entre as variáveis na representação gráfica do dendrograma, dá-se por meio da correlação de Pearson, sendo que, à medida que existe um maior grau de correlação entre as variáveis, menor será a distância entre as mesmas. Observa-se nas Tabela 7 e Tabela 8, a correlação de Pearson entre as variáveis analisadas para a cultura da soja em relação a cobertura vegetal de milheto, para o nível de significância de 5\%, para os anos 2013/14 e 2014/15 respectivamente. 
Tabela 2. Correlação de Pearson entre as variáveis de saída analisadas para a cultura da soja com cobertura vegetal de milheto para nível de significância de 5\% (Ano 2013/14).

\begin{tabular}{|c|c|c|c|c|c|c|c|c|c|c|c|c|c|c|c|c|c|}
\hline & PRO & EST & AIV & APL & NVP & NGP & NGV & MGR & $\mathbf{T N}$ & $\mathbf{T P}$ & TK & TCa & TMg & TS & TMo & $\mathbf{T C u}$ & TZn \\
\hline EST & - & & & & & & & & & & & & & & & & \\
\hline AIV & - & - & & & & & & & & & & & & & & & \\
\hline APL & - & - & - & & & & & & & & & & & & & & \\
\hline NVP & - & - & - & - & & & & & & & & & & & & & \\
\hline NGP & 0,737 & - & - & 0,287 & 0,292 & & & & & & & & & & & & \\
\hline NGV & 0,324 & - & - & - & $-0,716$ & 0,411 & & & & & & & & & & & \\
\hline MGR & 0,290 & - & - & 0,316 & 0,35 & 0,323 & - & & & & & & & & & & \\
\hline $\mathbf{T N}$ & - & - & - & - & - & - & - & - & & & & & & & & & \\
\hline $\mathbf{T P}$ & - & - & - & - & - & - & - & - & - & & & & & & & & \\
\hline TK & - & $-0,437$ & - & - & - & - & - & - & - & - & & & & & & & \\
\hline TCa & - & - & - & - & - & - & - & - & $-0,299$ & - & $-0,354$ & & & & & & \\
\hline TMg & - & 0,296 & - & - & - & - & - & - & $-0,294$ & - & - & 0,730 & & & & & \\
\hline TS & - & - & - & - & - & - & - & - & - & 0,606 & - & - & - & & & & \\
\hline TMo & - & - & - & - & - & - & - & - & - & - & - & - & - & - & & & \\
\hline $\mathbf{T C u}$ & - & - & - & - & - & - & - & - & - & - & - & $-0,361$ & $-0,404$ & - & - & & \\
\hline TZn & - & - & - & - & - & - & - & - & - & - & - & - & - & 0,288 & - & - & \\
\hline TMn & - & - & - & - & - & - & - & - & - & - & - & $-0,375$ & $-0,297$ & - & - & 0,445 & 0,387 \\
\hline
\end{tabular}


Tabela 3. Correlação de Pearson entre as variáveis de saída analisadas para a cultura da soja com cobertura vegetal de milheto para nível de significância de 5\% (Ano 2014/15).

\begin{tabular}{|c|c|c|c|c|c|c|c|c|c|c|c|c|c|c|c|c|c|}
\hline & PRO & EST & AIV & APL & NVP & NGP & NGV & MGR & TN & $\mathbf{T P}$ & TK & TCa & TMg & TS & TMo & $\mathbf{T C u}$ & TZn \\
\hline EST & - & & & & & & & & & & & & & & & & \\
\hline AIV & - & - & & & & & & & & & & & & & & & \\
\hline APL & - & 0,427 & - & & & & & & & & & & & & & & \\
\hline NVP & 0,319 & - & - & - & & & & & & & & & & & & & \\
\hline NGP & - & - & - & - & 0,569 & & & & & & & & & & & & \\
\hline NGV & - & - & - & - & - & 0,627 & & & & & & & & & & & \\
\hline MGR & - & - & - & $-0,365$ & - & - & - & & & & & & & & & & \\
\hline $\mathbf{T N}$ & - & $-0,287$ & - & - & - & - & - & 0,372 & & & & & & & & & \\
\hline $\mathbf{T P}$ & $-0,392$ & - & - & - & - & - & - & $-0,468$ & - & & & & & & & & \\
\hline TK & - & - & - & - & - & - & - & 0,329 & - & - & & & & & & & \\
\hline TCa & 0,295 & $-0,316$ & - & - & - & 0,393 & - & - & - & $-0,286$ & - & & & & & & \\
\hline TMg & - & $-0,353$ & - & $-0,301$ & - & - & - & 0,415 & 0,426 & $-0,296$ & - & 0,487 & & & & & \\
\hline TS & - & $-0,445$ & - & - & - & - & - & 0,388 & 0,500 & - & - & 0,300 & 0,668 & & & & \\
\hline TMo & - & - & - & - & $-0,356$ & - & 0,284 & - & - & - & - & - & - & - & & & \\
\hline $\mathbf{T C u}$ & - & - & - & - & - & - & - & - & - & - & - & - & - & - & - & & \\
\hline TZn & - & - & - & - & - & - & - & 0,311 & 0,447 & - & - & - & 0,422 & 0,503 & - & - & \\
\hline TMn & - & - & - & - & - & - & - & - & - & - & - & - & - & - & - & - & - \\
\hline
\end{tabular}

Legenda: Produtividade (PRO) [kg/ha], Estande (EST) [mil plantas/ha], Altura de inserção da primeira vagem (AIV) [cm], Altura de planta (APL) [cm], Número de vagens por planta (NVP) [unid.], Número de grãos por planta (NGP) [unid.], Número de grãos por vagem (NGV) [unid.], Massa de 1000 grãos (MGR) [unid.], Teor foliar de nitrogênio (TN) [g/kg], Teor foliar de fósforo (TP) [g/kg], Teor foliar de potássio (TK) [g/kg], Teor foliar de cálcio (TCa) $[\mathrm{g} / \mathrm{kg}$ ], Teor foliar de magnésio (TMg) $[\mathrm{g} / \mathrm{kg}$ ], Teor foliar de enxofre (TS) [g/kg], Teor foliar de molibdênio (TMo) [mg/kg], Teor foliar de cobre $(\mathrm{TCu})[\mathrm{mg} / \mathrm{kg}]$, Teor foliar de zinco (TZn) $[\mathrm{mg} / \mathrm{kg}]$, Teor foliar de manganês (TMn) $[\mathrm{mg} / \mathrm{kg}]$. Fonte: Elaborada pelos autores. 
Nota-se na Tabela 2, o alto grau de correlação entre as variáveis PRO e NGP, sendo demonstrada pela proximidade entre ambas, na análise gráfica do dendrograma (Figura 1); já por sua vez, a correlação negativa de 0,716 entre as variáveis NVP e NGV, explica o seu distanciamento gráfico, referente ao ano 2013/14, expressando resultados diferentes no experimento realizado de Marin, Santos e Babinot Junior (2018), que analisaram a produtividade da soja em relação a palhada de milho e braquiária.

Verifica-se por sua vez, a correlação negativa existente entre o Teor foliar de nitrogênio e as variáveis, Teor foliar de magnésio e Teor foliar de cálcio, sendo de $-0,299$ e $-0,294$ respectivamente, já por sua vez, nota-se entre $\mathrm{TMg}$ e $\mathrm{TCa}$, uma correlação positiva de 0,730 , corroborando com o resultado obtido por Kurihara et al. (2013) no trabalho sobre o acúmulo de nutrientes da soja, no qual, os nutrientes apresentaram a mesma tendência de comportamento.
Já para o segundo ciclo da cultura, verifica-se a correlação (Tabela 8) positiva entre as $\mathrm{TMg}$ e TS, demonstrada graficamente no dendrograma (Figura 2), sua proximidade em relação ao grau de similaridade de seus comportamentos. Já a dissimilaridade é notada pela correlação negativa existente entre a variável TP com as variáveis PRO e MGR, -0,392 e -0,468 respectivamente, assim como $\mathrm{O}$ distanciamento gráfico das variáveis.

Da mesma maneira, foi também realizada a análise multivariada para as 18 variáveis de saída da cultura da soja cultivada sob a palhada do sorgo durante os anos agrícolas 2013/14 e 2014/15, também com base na correlação de Pearson com nível de significância de 5\%.

Sendo assim, estão representados nas Figura 3 e Figura 4, a análise gráfica de dendrograma, os níveis de similaridade entre as variáveis de saída.

Figura 3. Dendrograma das variáveis de saída da cultura da soja, do ano agrícola 2013/14 para a cobertura vegetal de sorgo.

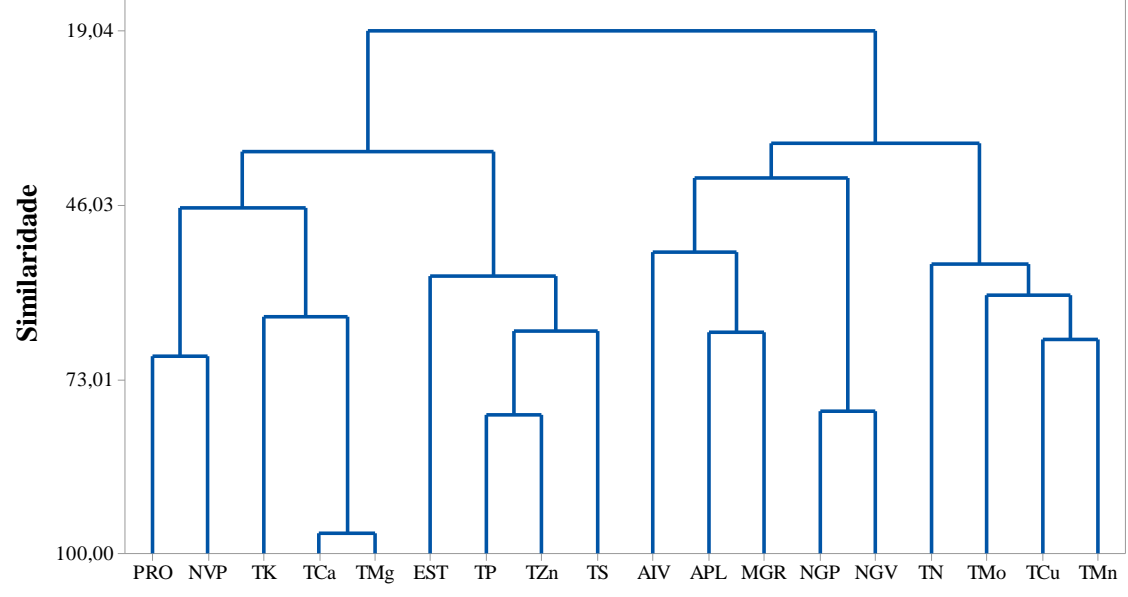

Fonte: Elaborada pelos autores. 
Figura 4. Dendrograma das variáveis de saída da cultura da soja, do ano agrícola 2014/15 para a cobertura vegetal de sorgo.

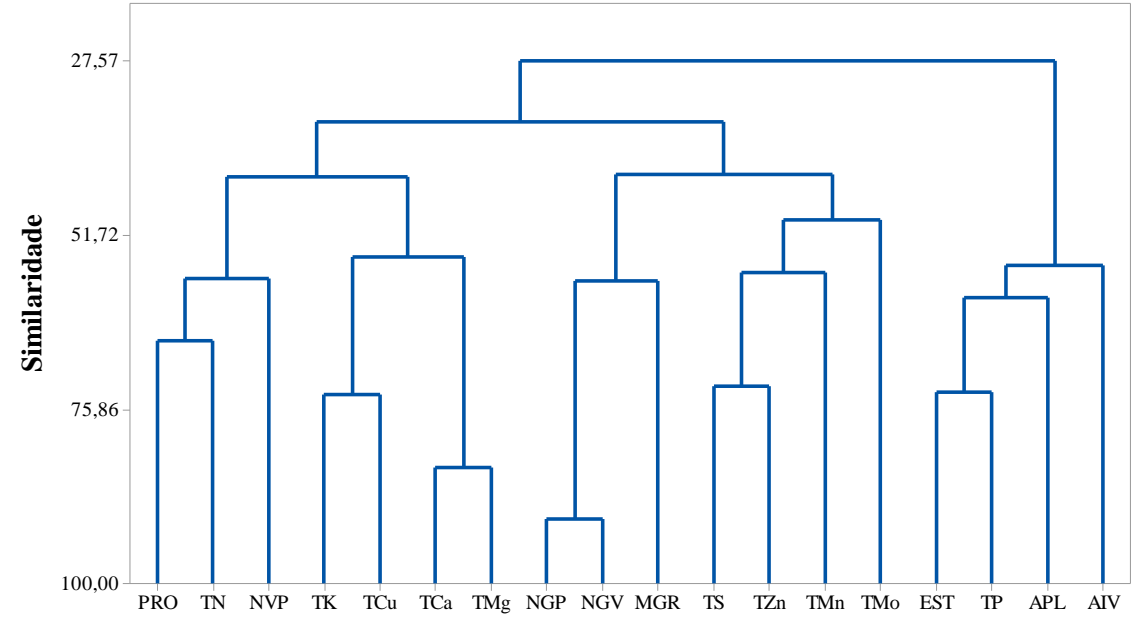

Fonte: Elaborada pelos autores.

O dendrograma representa o agrupamento das variáveis em grupos menores, denominados cluster, por meio do grau de similaridade entre as mesmas. Quanto maior a similaridade em relação ao comportamento das variáveis, menor será o número de agrupados, já por outro lado, haverá uma maior quantidade de variáveis em um mesmo cluster para um nível de similaridade menor.

Do mesmo modo como ocorrido para palhada do milheto, o teor de nitrogênio e potássio, tiveram comportamentos semelhantes em relação as variáveis de produtividade da soja, principalmente em relação ao segundo ano do experimento, corroborando com os resultados obtidos para a cobertura de milheto, podendo atribuir a influência desses nutrientes na produtividade da cultura.
Por sua vez, foi também observado comportamento semelhante das variáveis de produtividade, número de vagem por planta e massa de 1000 grãos, no experimento realizado por Silva et al. (2019), quando da soja cultivada sob diferentes doses de fertilizantes organomineral.

A composição gráfica do dendrograma é realizada com base nos passos de Amalgamação, que trazem consigo informações referentes à distância dos grupos formados em relação ao nível de similaridade entre as variáveis estudadas a cada passo, como pode-se observar na Tabela 4. 
Tabela 4. Passos de Amalgamação das variáveis de saída da cultura da soja para a cobertura vegetal de sorgo.

\begin{tabular}{c|ccc|c|crc}
\hline \multicolumn{5}{c|}{$\mathbf{2 0 1 3} / \mathbf{1 4}$} & \multicolumn{4}{|c}{$\mathbf{2 0 1 4 / 1 5}$} \\
\hline Passo & Agrupados & Similaridade & Distância & Passo & \multicolumn{4}{c}{ Agrupados } & Similaridade & Distância \\
\hline $\mathbf{1}$ & 17 & 96,7034 & 0,06593 & $\mathbf{1}$ & 17 & 90,9285 & 0,18143 \\
$\mathbf{2}$ & 16 & 78,4261 & 0,43148 & $\mathbf{2}$ & 16 & 83,8482 & 0,32304 \\
$\mathbf{3}$ & 15 & 77,81 & 0,44380 & $\mathbf{3}$ & 15 & 73,7568 & 0,52486 \\
$\mathbf{4}$ & 14 & 69,3329 & 0,61334 & $\mathbf{4}$ & 14 & 73,4934 & 0,53013 \\
$\mathbf{5}$ & 13 & 66,7245 & 0,66551 & $\mathbf{5}$ & 13 & 72,6614 & 0,54677 \\
$\mathbf{6}$ & 12 & 65,6171 & 0,68766 & $\mathbf{6}$ & 12 & 66,3537 & 0,67293 \\
$\mathbf{7}$ & 11 & 65,5334 & 0,68933 & $\mathbf{7}$ & 11 & 60,2952 & 0,79410 \\
$\mathbf{8}$ & 10 & 63,1962 & 0,73608 & $\mathbf{8}$ & 10 & 57,9801 & 0,84040 \\
$\mathbf{9}$ & 9 & 59,9443 & 0,80111 & $\mathbf{9}$ & 9 & 57,7145 & 0,84571 \\
$\mathbf{1 0}$ & 8 & 57,0434 & 0,85913 & $\mathbf{1 0}$ & 8 & 56,8138 & 0,86372 \\
$\mathbf{1 1}$ & 7 & 55,0777 & 0,89845 & $\mathbf{1 1}$ & 7 & 55,9009 & 0,88198 \\
$\mathbf{1 2}$ & 6 & 53,2064 & 0,93587 & $\mathbf{1 2}$ & 6 & 54,6846 & 0,90631 \\
$\mathbf{1 3}$ & 5 & 46,3773 & 1,07245 & $\mathbf{1 3}$ & 5 & 49,5937 & 1,00813 \\
$\mathbf{1 4}$ & 4 & 41,8703 & 1,16259 & $\mathbf{1 4}$ & 4 & 43,6396 & 1,12721 \\
$\mathbf{1 5}$ & 3 & 37,7123 & 1,24575 & $\mathbf{1 5}$ & 3 & 43,2371 & 1,13526 \\
$\mathbf{1 6}$ & 2 & 36,4028 & 1,27194 & $\mathbf{1 6}$ & 2 & 35,9701 & 1,28060 \\
$\mathbf{1 7}$ & 1 & 19,0417 & 1,61917 & $\mathbf{1 7}$ & 1 & 27,5747 & 1,44851 \\
\hline
\end{tabular}

Fonte: Elaborada pelos autores.

Com base na Tabela 4, é possível verificar a formação distinta de 2 grupos concentrados no primeiro ano e 3 grupos para o segundo ano, uma vez que ocorre um aumento abrupto da distância em relação ao aumento do passo de amalgamação, sendo 16 para o 17 e 15 para 16, no 2013/2014 e 2014/2015, respectivamente. 
Tabela 5. Correlação de Pearson entre as variáveis de saída analisadas para a cultura da soja com cobertura vegetal de sorgo para nível de significância de 5\% (Ano 2013/14).

\begin{tabular}{|c|c|c|c|c|c|c|c|c|c|c|c|c|c|c|c|c|c|}
\hline & PRO & EST & AIV & APL & NVP & NGP & NGV & MGR & TN & $\mathbf{T P}$ & TK & TCa & TMg & TS & TMo & TCu & TZn \\
\hline EST & - & & & & & & & & & & & & & & & & \\
\hline AIV & $-0,286$ & $-0,318$ & & & & & & & & & & & & & & & \\
\hline APL & - & - & - & & & & & & & & & & & & & & \\
\hline NVP & 0,387 & - & $-0,303$ & $-0,162$ & & & & & & & & & & & & & \\
\hline NGP & 0,451 & - & - & - & - & & & & & & & & & & & & \\
\hline NGV & - & - & - & - & $-0,619$ & 0,556 & & & & & & & & & & & \\
\hline MGR & - & - & - & 0,312 & - & - & - & & & & & & & & & & \\
\hline $\mathbf{T N}$ & - & - & - & - & - & - & - & - & & & & & & & & & \\
\hline $\mathbf{T P}$ & - & - & - & - & - & - & - & - & - & & & & & & & & \\
\hline TK & - & - & - & - & - & - & - & - & - & - & & & & & & & \\
\hline TCa & - & - & - & - & - & - & - & - & - & - & - & & & & & & \\
\hline TMg & - & - & - & - & - & - & - & - & - & - & 0,413 & 0,934 & & & & & \\
\hline TS & - & - & - & - & - & $-0,285$ & - & - & - & 0,541 & - & - & - & & & & \\
\hline TMo & - & - & - & - & - & - & - & - & - & - & - & - & - & - & & & \\
\hline $\mathbf{T C u}$ & - & - & - & - & - & - & - & - & 0,318 & 0,293 & - & $-0,307$ & - & - & - & & \\
\hline TZn & - & - & - & - & - & - & - & - & - & 0,569 & - & - & - & 0,311 & - & - & \\
\hline TMn & - & - & - & - & - & - & - & - & - & - & - & - & - & - & - & 0,334 & - \\
\hline
\end{tabular}

Legenda: Produtividade (PRO) [kg/ha], Estande (EST) [mil plantas/ha], Altura de inserção da primeira vagem (AIV) [cm], Altura de planta (APL) [cm], Número de vagens por planta (NVP) [unid.], Número de grãos por planta (NGP) [unid.], Número de grãos por vagem (NGV) [unid.], Massa de 1000 grãos (MGR) [unid.], Teor foliar de nitrogênio (TN) [g/kg], Teor foliar de fósforo (TP) [g/kg], Teor foliar de potássio (TK) [g/kg], Teor foliar de cálcio (TCa) $[\mathrm{g} / \mathrm{kg}$ ], Teor foliar de magnésio (TMg) $[\mathrm{g} / \mathrm{kg}$ ], Teor foliar de enxofre (TS) [g/kg], Teor foliar de molibdênio (TMo) [mg/kg], Teor foliar de cobre $(\mathrm{TCu})[\mathrm{mg} / \mathrm{kg}]$, Teor foliar de zinco (TZn) $[\mathrm{mg} / \mathrm{kg}]$, Teor foliar de manganês (TMn) $[\mathrm{mg} / \mathrm{kg}]$. Fonte: Elaborada pelos autores. 
Tabela 6. Correlação de Pearson entre as variáveis de saída analisadas para a cultura da soja com cobertura vegetal de sorgo para nível de significância de 5\% (Ano 2014/15).

\begin{tabular}{|c|c|c|c|c|c|c|c|c|c|c|c|c|c|c|c|c|c|}
\hline & PRO & EST & AIV & APL & NVP & NGP & NGV & MGR & TN & $\mathbf{T P}$ & TK & TCa & TMg & TS & TMo & $\mathbf{T C u}$ & TZn \\
\hline EST & - & & & & & & & & & & & & & & & & \\
\hline AIV & - & 0,397 & & & & & & & & & & & & & & & \\
\hline APL & - & 0,467 & - & & & & & & & & & & & & & & \\
\hline NVP & - & - & - & - & & & & & & & & & & & & & \\
\hline NGP & - & - & $-0,3$ & - & 0,318 & & & & & & & & & & & & \\
\hline NGV & - & - & $-0,309$ & - & - & 0,819 & & & & & & & & & & & \\
\hline MGR & - & - & - & - & - & - & - & & & & & & & & & & \\
\hline $\mathbf{T N}$ & 0,327 & - & - & - & - & - & - & - & & & & & & & & & \\
\hline $\mathbf{T P}$ & - & 0,47 & - & - & - & - & - & - & 0,29 & & & & & & & & \\
\hline TK & 0,352 & - & - & - & - & - & - & - & - & - & & & & & & & \\
\hline TCa & - & $-0,36$ & - & - & - & - & - & 0,37 & - & - & - & & & & & & \\
\hline TMg & - & $-0,449$ & - & $-0,326$ & - & - & - & - & - & $-0,371$ & 0,312 & 0,677 & & & & & \\
\hline TS & - & - & - & - & - & - & - & - & - & - & - & 0,405 & 0,299 & & & & \\
\hline TMo & - & - & - & - & - & - & - & - & - & - & - & - & - & - & & & \\
\hline $\mathbf{T C u}$ & - & - & - & - & - & - & - & - & - & - & 0,475 & - & 0,288 & - & - & & \\
\hline TZn & - & - & - & - & - & - & - & - & 0,368 & - & - & - & - & 0,453 & - & - & \\
\hline TMn & - & - & - & - & - & - & - & - & 0,285 & - & - & - & - & - & - & - & - \\
\hline
\end{tabular}

Legenda: Produtividade (PRO) [kg/ha], Estande (EST) [mil plantas/ha], Altura de inserção da primeira vagem (AIV) [cm], Altura de planta (APL) [cm], Número de vagens por planta (NVP) [unid.], Número de grãos por planta (NGP) [unid.], Número de grãos por vagem (NGV) [unid.], Massa de 1000 grãos (MGR) [unid.], Teor foliar de nitrogênio (TN) [g/kg], Teor foliar de fósforo (TP) [g/kg], Teor foliar de potássio (TK) [g/kg], Teor foliar de cálcio (TCa) $[\mathrm{g} / \mathrm{kg}$ ], Teor foliar de magnésio (TMg) $[\mathrm{g} / \mathrm{kg}$ ], Teor foliar de enxofre (TS) [g/kg], Teor foliar de molibdênio (TMo) [mg/kg], Teor foliar de cobre $(\mathrm{TCu})[\mathrm{mg} / \mathrm{kg}]$, Teor foliar de zinco (TZn) $[\mathrm{mg} / \mathrm{kg}]$, Teor foliar de manganês (TMn) $[\mathrm{mg} / \mathrm{kg}]$. Fonte: Elaborada pelos autores. 
De mesmo modo, o comportamento das variáveis apresentadas nos dendrogramas da Figura 3 e Figura 4, é reflexo da análise de correlação entre as mesmas, para as quais, aproximam-se as variáveis que possuem maior grau da correlação de Pearson, e distanciam-se a medida em que o mesmo diminui.

Nota-se no 2013/2014 1 (Figura 3) o alto grau de similaridade entre as variáveis

\section{CONCLUSÕES}

Foi possível verificar a formação de poucos clusters para ambas coberturas vegetais entre as variáveis analisadas para cultura da soja cultivada sob sistema plantio direto.

Desse modo, constatou-se uma grande proximidade entre as variáveis

\section{REFERÊNCIAS BIBLIOGRÁFICAS}

ARATANI; R. G.; MADALOSSO, G.; POMPEU, A. T. Percepção e prática do sistema plantio direto por agricultores mato-grossenses. Revista de Ciências Agroambientais, v.16, n.1, p.1-7, 2018.

BERNARDES, J. V. S.; ORIOLI JÚNIOR, V.; ARAUJO, J. P. N. Aplicação foliar de molibdênio não influencia a produtividade de soja em solo com acidez corrigida.

Revista Inova Ciência \& Tecnologia, v. 5, n. 2, p. 12-17, 2019.

BIESDORF, E. M. et al. Efeito inibitório do sorgo granífero na cultura da soja semeada em sucessão. Revista Brasileira de Milho e Sorgo, v.17, n.3, p. 445-459, 2018.

CENTURION, J. F. Balanço hídrico na região de Ilha Solteira. Científica, v.10, n.1, p. 57-61, 1982.

CHANDRASHEKAR, G.; SAHIN, F. A Survey on Feature Selection Methods. Computers \& Electrical Engineering, v.40, n.1, p. 16-28, 2014.
TCa e TMg, para a qual possuem o valor de 0,934 da correlação de Pearson, por outro lado, o comportamento oposto é verificado entre as variáveis EST e $\mathrm{TMg}$ durante o Ano 2, com grau de correlação de $-0,449$, refletindo no distanciamento gráfico apresentado no dendrograma da Figura 4.

indicadoras de produtividade da cultura, com as variáveis de teores dos nutrientes de nitrogênio e potássio, evidenciada por uma forte correlação entre as mesmas.

CHONG, C. Y.; LEE, S. P.; LING, T. C. Efficient Software Clustering Technique Using an Adaptive and Preventive Dendrogram Cutting Approach.

Information and Software Technology, v.55, n.11, p. 1994-2012, 2013.

FAGUNDES, M. O. et al. Qualidade de um latossolo sob plantio convencional e sistema plantio direto no cerrado baiano, Brasil. Revista Ibero Americana de Ciências Ambientais, v.10, n.3, p.281297, 2019.

FORINA, M. et al. A New Algorithm for Seriation and Its Use in Similarity Dendrograms. Chemometrics and Intelligent Laboratory Systems, v.87, n.2, p. 262-274, 2007.

GOES, R. J. Doses de Nitrogênio em Coberturas Vegetais e Molibdênio Foliar na Soja em Sucessão. 2016. 77 f. Tese (Doutorado) - Pós-Graduação em Agronomia (Sistemas de Produção), Universidade Estadual Paulista, Ilha Solteira, 2016. 
HONGYU, K., SANDANIELO, V. L. M., JUNIOR, G. J. O. Análise de Componentes Principais: resumo teórico, aplicação e interpretação. Engineering and Science, v.5, n.1, p.83-90, 2016.

KURIHARA, C. H. et al. Acúmulo de matéria seca e nutrientes em soja, como variável do potencial produtivo. Revista Ceres, v.60, n.5, p. 690-698, 2013.

LOPES, I. et al. Análise multivariada no estudo da variação do Índice de Aridez da Bahia e Pernambuco, Agrometeoros, v.26, n.1, p.93-102, 2018.

MARIN, C. M.; SANTOS, E. L.; BALBINOT JUNIOR, A. A. Produtividade e componentes de rendimento da soja em função da quantidade de palha de milho e braquiária. In: VIII Congresso Brasileiro de Soja, Goiânia-GO, p. 377-379, 2018.

PARDO, G.; MARTÍNEZ, Y.

Conservation agriculture in trouble? Estimating the economic impact of an eventual glyphosate in spain. Planta Daninha, v.37, 2019.

PASSOS, A. M. A. et al. Cultivares de soja em sucessão ao trigo nos sistemas convencional e plantio direto. Revista Agrarian, v.8, n.27, p.30-38, 2015.

PEREIRA, C. S. et al. Doses de potássio com a presença de enxofre na cultura da soja. Global Science and Technology, v.9, n.1, p.22-32, 2016.

RAIJ, B. V. et al. Recomendações de adubação e calagem para o Estado de São Paulo. Instituto Agronômico - IAC, (IAC. Boletim Técnico, 100), v.1, n.2, p. 285287, 1996.

ROSSI, C. Q. et al. Decomposição e liberação de nutrientes da palhada de braquiária, sorgo e soja em áreas de plantio direto no cerrado goiano. Seminário:

Ciências Agrárias, v. 34, n. 4, p. 15231534, 2013.
SANTOS, F.; CAMPOS, C. S. S. O avanço da sojicultura no nordeste brasileiro: reflexões iniciais sobre a região da SEALBA. Diversitas Journal, v.5, n.1, p.203-220, 2020.

SANTOS, H. G. et al. Sistema Brasileiro de Classificação de Solos. 5. ed. Brasília, DF: Embrapa, 2018.

ALMEIDA JÚNIOR et al. Analise das variáveis tecnologicas na cultura da soja (glycine max) com utilização de remineralizador de solo como fertilizante.

Brazilian Journal of Development, v.6, n. 8, p. 56835-56847, 2020.

SCHNEIDER, F. et al. Eficiência agronômica da cultura da soja (Glycine max (L.) Merril) submetida a coinoculação. Revista Scientia Agraria, v. 18, n. 4, p. 72-79, 2017.

SILVA, L.L.; NETO, N. Análise de eficiência de diferentes fungicidas no controle do fungo Phakopsora Pachyrhizi na cultura da soja. Ciência \& Tecnologia, v. 3, n. 1, p. 44-51, 2019

SILVA, M. L. et al. Padrão de especialização do comércio internacional agrícola brasileiro: uma análise por meio de indicadores de competitividade. Revista em Agronegócio e Meio Ambiente, v. 11, n. 2, p. 385-408, 2018.

SILVA, M. P. et al. Plantas de cobertura e qualidade química e física de Latossolo Vermelho distrófico sob plantio direto. Agrária - Revista Brasileira de Ciências Agrárias, v.12, n.1, p.60-67, 2017.

SILVA, V. J. A. Análise das variáveis tecnológicas da soja em função das doses crescentes de fertilizantes organomineral. In: IV Colóquio Estadual de Pesquisa Multidisciplinar e II Congresso Nacional de Pesquisa Multidisciplinar, p. 1-6, 2019. 
STRECK, E. A. et al. Variabilidade fenotípica de genótipos de arroz irrigado via análise multivariada. Revista Ciência Agronômica, v. 48, n. 1, p. 101-109, 2017.

VITORINO, R. F. Características

fisiológicas e biométricas de plantas de soja tratadas com fitorreguladores e bioestimulantes de crescimento. 2014. 37 f. Dissertação (Mestrado) - Pós-Graduação em Ciências Agrárias (Produção Vegetal), Instituto Federal Goiano, Rio Verde, 2014. 